\title{
Evaluation of Angiogenesis in Multiple Myeloma by VEGF Immunoexpression and Microvessel Density
}

\author{
Anshu Palta ${ }^{1}$ Manveen Kaur ${ }^{1}$ Anita Tahlan ${ }^{1}$ Kislay Dimri \\ ${ }^{1}$ Department of Pathology, Government Medical College and \\ Hospital, Chandigarh, India \\ 2Department of Radiation Oncology, Government Medical College

\begin{abstract}
Address for correspondence Manveen Kaur, MD, Department of Pathology, Government Medical College and Hospital, Chandigarh 160031, India (e-mail: docmanveen@gmail.com).
\end{abstract} and Hospital, Chandigarh, India

J Lab Physicians:2020;12:38-43

\begin{abstract}
Background Multiple myeloma (MM) is a plasma cell disorder characterized by monoclonal proliferation of plasma cells in bone marrow. Plasmablastic MM is a morphologic subset of $\mathrm{MM}$, containing $\geq 2 \%$ plasmablasts of all plasma cells.

Methods The study included 30 consecutively diagnosed patients of MM (6 plasmablastic, 24 nonplasmablastic) over a span of 2 years. Angiogenesis in MM was assessed by analysis of vascular endothelial growth factor (VEGF) immunoexpression by plasma cells and microvessel density (MVD) using anti-CD34 antibody. CD34 and VEGF immunohistochemical staining was performed in all the 30 cases. Angiogenesis was studied in relation to plasmablastic morphology and clinical profile to determine if any correlation exists between these.

Results The mean VEGF expression of $80.83 \pm 7.36$ in plasmablastic myeloma cases was significantly higher compared with a mean VEGF of $53.54 \pm 17.09$ in nonplasmablastic cases. Most of the cases (66.6\%) of plasmablastic myeloma exhibited strong (3+) VEGF expression. The difference in mean VEGF expression between plasmablastic and nonplasmablastic cases was found to be statistically significant $(p=0.001)$. The mean MVD in plasmablastic cases was $44.8 \pm 3.69$, while in the nonplasmablastic category, the mean MVD was $23.7 \pm 5.14$, difference being statistically significant $(p<0.05)$.

Keywords

- multiple myeloma

- plasmablastic

- angiogenesis

- microvessel density

- vascular endothelial growth factor

Also, a positive correlation was found between VEGF expression and MVD.

Conclusion A moderate/strong VEGF intensity and higher MVD were found in cases of plasmablastic MM, suggesting that a more aggressive histological disease may be associated with increased production of VEGF. This finding might be helpful to identify a subset of patients with adverse prognosis and to provide antiangiogenic therapy to improve their survival. However, studies comprising larger number of patients are required to bring out a statistical significance to further substantiate these findings.
\end{abstract}

\section{Introduction}

Multiple myeloma (MM) is a plasma cell disorder accounting for 10 to $15 \%$ of all hematopoietic neoplasms. It is characterized by monoclonal proliferation of plasma cells in bone marrow producing serum or urinary $M$ protein, lytic lesions in bone, and various clinical and laboratory abnormalities. ${ }^{1}$ It is a progressive disease with an extremely poor outcome. Plasmablastic MM is a morphologic subset of $\mathrm{MM}$, containing $\geq 2 \%$ plasmablasts of all the plasma cells, accounting for $\sim 20 \%$ of all myeloma cases. ${ }^{2}$

Many studies have provided insights into the pathogenesis of this disease emphasizing on defects involving proliferation, apoptosis, and angiogenesis. ${ }^{3}$ Angiogenesis refers published online July 24,2020
DOI https://doi.org/

10.1055/s-0040-1714933 ISSN 0974-2727.
(C)2020 by The Indian Association of Laboratory Physicians
License terms

() (1) $\odot \circledast$ 
to the formation of new blood vessels that is important for the proliferation of most of the malignant neoplasms. The amount of microvessels in the bone marrow progressively increases along the spectrum of plasma cell disorders from monoclonal gammopathy of undetermined significance to smoldering MM to MM. ${ }^{4}$ Angiogenic cytokines such as vascular endothelial growth factor (VEGF), hepatocyte growth factor, and basic fibroblast growth factor are overexpressed in MM cell lines. ${ }^{5}$ Various techniques have been developed for visualizing and estimating the number of these microvessels. Immunohistochemical staining for endothelial markers such as CD34, von Willebrand factor, and CD31 allows excellent visualization of the microvessels. Angiogenesis can then be quantified in terms of the microvessel density (MVD). ${ }^{6}$

While the literature is replete with studies on angiogenesis in MM, and its correlation with the prognosis, there is paucity of studies on correlation between the morphology of plasma cells, MVD, and VEGF immunoexpression. The present study aims to assess angiogenesis in MM by immunohistochemical analysis of VEGF expression by the plasma cells and MVD by anti-CD34 antibody. Correlation between VEGF and MVD has been studied in detail to provide valuable information in the pathogenesis of myeloma. Angiogenesis has been studied in relation to the morphologic features (especially plasmablastic morphology) and clinical profile to determine if any correlation exists between these.

\section{Materials and Methods}

This a retrospective study including 30 consecutively diagnosed patients of MM (6 plasmablastic, 24 nonplasmablastic) based on clinical, radiological, biochemical, electrophoresis, and bone marrow findings, over a span of 2 years. All the patients with a final diagnosis of MM and irrespective of age and gender were included. Patients who had received prior chemotherapy were excluded from the study.

Detailed clinical data, biochemical and radiological features, plates of agarose gel electrophoresis, hemogram findings, and slides of bone marrow aspirate and trephine biopsies were retrieved from archival material. The percentage of plasma cells (tumor burden) was counted and the disease was classified as plasmablastic or nonplasmablastic according to the Greipp et al. ${ }^{2}$

CD34 and VEGF immunohistochemical staining were performed in all the 30 cases. After deparaffinization in xylene, the sections were rehydrated through series of graded alcohol (70, 90, and 100\%) and distilled water. Endogenous blocking was done using $3 \%$ hydrogen peroxide in methanol. Antigen retrieval was performed in pressure cooker in citrate buffer (pH 6.0). Then sections were incubated overnight with primary antibodies CD34 and VEGF in a moist chamber. Slides were brought to room temperature and washed with Tris buffer. Sections were then covered with secondary antibody for 30 minutes and then again washed with Tris buffer. Chromogen (DAB) was added onto slides for 1 to 3 minutes and checked under a microscope. Counterstaining was done with Cole's hematoxylin for 1 minute.

\section{Evaluation of VEGF Expression and Microvessel Density VEGF Expression}

VEGF immunostained slides were examined for the cytoplasmic expression of VEGF (both qualitative and quantitative) in the plasma cells. The percentage of positively staining plasma cells was assessed. If the number was greater than $10 \%$, VEGF expression was graded subjectively according to the intensity of staining as weak (1+), moderate (2+), and strong (3+). $\mathrm{H}$-score was calculated using the equation listed below:

$$
\mathrm{H} \text { score }=\text { intensity of staining } \times \% \text { positivity }
$$

Megakaryocytes exhibit a strong VEGF expression; hence, they were used as positive internal control. ${ }^{7}$

\section{Microvessel Density}

The vascular endothelium was highlighted using CD34 immunostaining. Angiogenesis was measured by counting the CD34-labeled microvessels (MVD). Any immunohistochemically stained endothelial cell cluster with or without lumen, clearly separate from adjacent microvessels, was counted as a distinct microvessel. Larger vessels with a thick muscular wall were excluded from the count.

The section was scanned at $100 \times$ magnification to locate the areas of high concentration of staining (hotspots). The microvessels were counted under 400× magnification in five hotspot areas and the MVD was reported as the average number of vessels in five hotspots. Counting was done in such a way that there was no repetition of the same hotspot/area. Patients were stratified into high angiogenesis (MVD > 20), intermediate angiogenesis (MVD 11-20), and low angiogenesis status (MVD < 10) according to previous studies. ${ }^{8-10}$ Ten bone marrow biopsies from nonmyeloma cases were taken as controls.

VEGF expression in the plasma cells was correlated with MVD in the bone marrow for assessing the role of VEGF as a potent angiogenic mediator in MM. In addition, correlation was also drawn between angiogenesis and the clinical profile of the patients, and with plasmablastic morphology of plasma cells.

\section{Statistical Analysis}

The data was analyzed using the SPSS software. Analysis of variance was used to test the differences in the studied parameters between plasmablastic and nonplasmablastic myeloma. A p-value $<0.05$ was considered significant. Pearson's correlation coefficient test was used to correlate VEGF immunoexpression with MVD.

\section{Results}

Age of the patients ranged from 40 to 83 years with a mean age of $59.5 \pm 10.32$ years. Most of the patients were in the sixth to seventh decade of life. Out of the 30 patients, 20 were males with male to female ratio of 1.5:1. The mean age of patients with plasmablastic myeloma was $64.83 \pm 10.22$ years, while in the nonplasmablastic category, 
the mean age was $58.17 \pm 10.09$ years. All the patients in the plasmablastic group were males. The most common presenting complaints included backache (56.6\%), followed by generalized weakness (16.6\%), and loss of weight and appetite (13.3\%). Six of the patients (20\%) had underlying chronic kidney disease. Four patients (13.3\%) presented with concomitant extramedullary plasmacytoma.

Results of biochemical analysis revealed hypercalcemia in six $(20 \%)$ patients. Elevated serum creatinine was observed in $12(40 \%)$ patients, while 15 out of 30 patients (50\%) had hypoalbuminemia. Serum $\beta 2$ microglobulin was raised in all the patients (normal: 1500-2200 ng/mL). Skeletal survey showed presence of lytic bony lesions in 13 out of 30 cases (43.3\%), including five cases (83.3\%) of plasmablastic MM and eight cases (33.3\%) of nonplasmablastic MM.

On serum electrophoresis, all patients (100\%) showed a positive "M" band in $\gamma$ globulin region. Clinically, a diagnosis of MM was considered as the only/one of the differential diagnoses in 27 cases (90\%), while in 3 patients (10\%), a diagnosis of MM was not suspected. The clinical diagnoses in these patients were myelodysplastic syndrome/pure red cell aplasia, aplastic anemia, and metastasis, respectively.

Hemogram revealed anemia in 29 patients (96.6\%), and thrombocytopenia in 6 out of $30(20 \%)$ patients, while 2 patients (6.7\%) presented with pancytopenia. Plasma cells in the peripheral blood $(\mathrm{PB})$ were found in five patients (16.6\%), absolute plasma cell count ranging from 90 to $980 / \mu \mathrm{L}$. No case of plasma cell leukemia was diagnosed. - Table 1 summarizes the clinical and laboratory features of the patients.

\section{Bone Marrow Findings}

The number of plasma cells on bone marrow aspiration ranged from 20 to $81 \%$ with a mean of $47.23 \pm 21.12$. Out of the 30 cases, $6(20 \%)$ were classified as plasmablastic myeloma. The percentage of bone marrow plasma cells (BMPCs) ranged from 39 to $75 \%$ in plasmablastic myeloma with a mean of $62.33 \pm 13.92$. In the nonplasmablastic category, the mean BMPCs were $43.46 \pm 21.1$, the difference being statistically significant $(p=0.04)$.

Additional findings observed on bone marrow aspiration included presence of benign histiocytes and pseudo-Gaucher cells (6.7\%), and increased iron stores (13.3\%). Additional findings on trephine biopsies were an increase in reticulin fibrosis in four cases (13.3\%), gelatinous transformation in two cases (6.7\%), fibrinoid necrosis in one case (3.3\%), and presence of lymphoid aggregates in two cases (6.7\%). The comparison of biochemical and hematologic profile of patients with plasmablastic and nonplasmablastic myeloma is shown in - Table 2 .

\section{Analysis of Angiogenesis}

VEGF immunoexpression was observed in all the 30 cases, ranging from 10 to $95 \%$, with a weak (1+), moderate (2+), and strong staining in 4 (13.3\%), 16 (53.3\%), and 10 (33.3\%) cases, respectively (-Fig. 1). Mean VEGF expression in plasmablastic myeloma cases $(n=6)$ was $80.83 \pm 7.36$ compared with a mean VEGF of $53.54 \pm 17.09$ in nonplasmablastic cases $(n=24)$. The difference in mean VEGF expression between plasmablastic and nonplasmablastic cases was found to be statistically significant $(p=0.001)$. Also, the intensity of VEGF immunoexpression was higher in plasmablastic myeloma, with four $(66.6 \%)$ and two (33.3\%) cases exhibiting a strong (3+) and moderate expression (2+), respectively. None of the cases of plasmablastic myeloma showed weak

Table 1 Clinical and laboratory features of the patients $(n=30)$

\begin{tabular}{|l|l|l|}
\hline \multicolumn{2}{|c|}{ Clinical and laboratory features } & $\begin{array}{l}\text { Number of cases } \\
(\%), n=30\end{array}$ \\
\hline \multirow{4}{*}{$\begin{array}{l}\text { Presenting } \\
\text { symptoms }\end{array}$} & Backache & $17(56.6 \%)$ \\
\cline { 2 - 3 } & Weakness & $05(16.6 \%)$ \\
\cline { 2 - 3 } & $\begin{array}{l}\text { Loss of weight and } \\
\text { appetite }\end{array}$ & $04(13.3 \%)$ \\
\cline { 2 - 3 } & Plasmacytoma & $04(13.3 \%)$ \\
\cline { 2 - 3 } & Refractory anemia & $1(3.3 \%)$ \\
\cline { 2 - 3 } & $\begin{array}{l}\text { Hematuria and } \\
\text { gum bleed }\end{array}$ & $1(3.3 \%)$ \\
\cline { 2 - 3 } & Macroglossia & $1(3.3 \%)$ \\
\cline { 2 - 3 } & Paraparesis & $1(3.3 \%)$ \\
\hline \multirow{5}{*}{ Laboratory } & Anemia & $29(96.6 \%)$ \\
\cline { 2 - 3 } & Thrombocytopenia & $06(20 \%)$ \\
\cline { 2 - 3 } & Pancytopenia & 02 \\
\cline { 2 - 3 } & $\begin{array}{l}\text { Peripheral blood } \\
\text { plasma cells }\end{array}$ & $05(16.6 \%)$ \\
\cline { 2 - 3 } & Hypercalcemia & $06(20 \%)$ \\
\hline & Hypoalbuminemia & $15(50 \%)$ \\
\hline & $\begin{array}{l}\text { Raised serum } \\
\text { creatinine }\end{array}$ & $12(40 \%)$ \\
\hline
\end{tabular}

Table 2 Comparison of biochemical and hematological findings between plasmablastic and nonplasmablastic MM

\begin{tabular}{|l|l|l|l|l|}
\hline & $\begin{array}{l}\text { Plasmablastic MM } \\
(\boldsymbol{n}=\mathbf{6})\end{array}$ & $\begin{array}{l}\text { Nonplasmablastic } \\
\text { MM }(\boldsymbol{n}=\mathbf{2 4})\end{array}$ & $\boldsymbol{p}$-Value & Significance \\
\hline Serum calcium & $11.4 \pm 2.10$ & $10.49 \pm 1.43$ & 0.216 & Not significant \\
\hline Serum creatinine & $4.93 \pm 3.87$ & $2.01 \pm 1.45$ & 0.005 & Significant \\
\hline Serum albumin & $3.23 \pm 0.43$ & $3.59 \pm 0.74$ & 0.274 & Not significant \\
\hline$\beta 2$ microglobulin & $8954.50 \pm 2839.637$ & $7221.46 \pm 3333.565$ & 0.57 & Not significant \\
\hline Average hemoglobin $(\mathrm{g} / \mathrm{dL})$ & 7.1 & 8.7 & 0.067 & Not significant \\
\hline BMPCs & 62.33 & 43.4 & 0.048 & Significant \\
\hline
\end{tabular}

Abbreviations: BMPCs, bone marrow plasma cells; MM, multiple myeloma. 


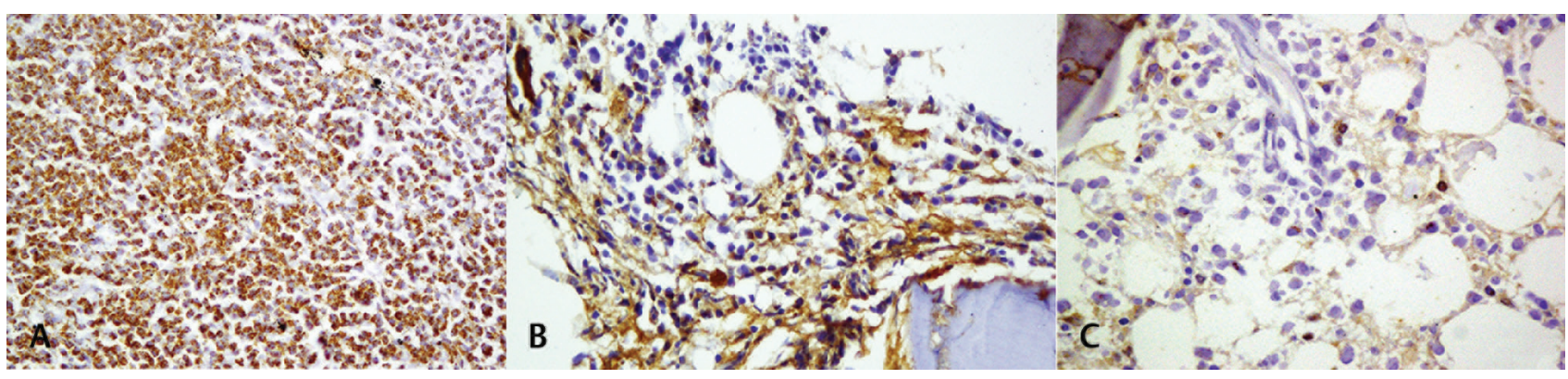

Fig. 1 Vascular endothelial growth factor (VEGF) immunoexpression by plasma cells. (A) Strong cytoplasmic VEGF expression (3+) in a case of plasmablastic multiple myeloma (MM) (400×). (B) Moderate-to-strong (2-3+) cytoplasmic VEGF expression in 50\% plasma cells (400×). Megakaryocytes used as internal control. (C) Weak (1+) cytoplasmic expression of VEGF $10 \%$ plasma cells in a case of nonplasmablastic MM (400×).

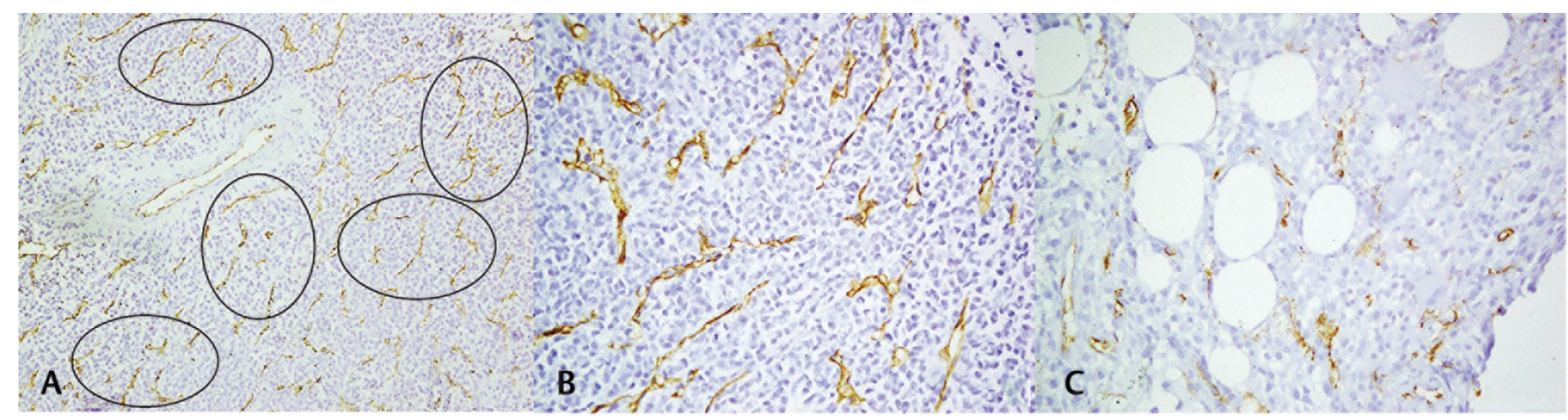

Fig. 2 Calculation of microvessel density (MVD) in multiple myeloma (MM). (A) Selection of hotspots in a section stained with CD34 antibody to highlight blood vessels (100×). (B) High MVD (> 20) in a case of plasmablastic MM (400×). (C) Intermediate MVD (11-20) in a case of nonplasmablastic MM (400×).

Table 3 Comparison of VEGF immunoexpression and MVD between plasmablastic and nonplasmablastic MM

\begin{tabular}{|l|l|l|l|l|l|}
\hline \multirow{5}{*}{ VEGF } & Weak (1+) & $\begin{array}{l}\text { Plasmablastic MM } \\
(\boldsymbol{n}=6)\end{array}$ & $\begin{array}{l}\text { Nonplasmablastic } \\
\text { MM }(\boldsymbol{n}=24)\end{array}$ & -Value & Significance \\
\cline { 2 - 6 } & Moderate (2+) & 0 & $4 / 24(16.7 \%)$ & \\
\cline { 2 - 6 } & Strong (3+) & $4 / 6(33.3 \%)$ & $14 / 24(58.3 \%)$ & & Significant \\
\hline & Mean & $80.83 \pm 7.36$ & $6 / 24(25 \%)$ & & 0.001 \\
\hline
\end{tabular}

Abbreviations: MM, multiple myeloma; MVD, microvessel density; VEGF, vascular endothelial growth factor.

VEGF immunoexpression. Average H-score in plasmablastic myeloma was 214.16, which was higher as compared to H-score of 119.16 in nonplasmablastic myeloma.

MVD was calculated as the average number of vessels in the five hotspots. Out of the 30 cases, 25 cases (83.3\%) exhibited high angiogenesis (MVD > 20), and five cases (16.6\%) exhibited intermediate angiogenesis (MVD 11-20) (-Fig. 2), while none of the patients had an MVD less than 10, as compared with controls who had MVD $<10$ (7.43 \pm 1.55$)$.

The mean MVD in plasmablastic cases was $44.8 \pm 3.69$, while in the nonplasmablastic category, the mean MVD was $23.7 \pm 5.14$, and this difference was found to be statistically significant $(p<0.05)$. - Table 3 shows comparison of VEGF immunoexpression and MVD between plasmablastic and nonplasmablastic MM. A positive correlation was found between VEGF expression and MVD (-Fig. 3).

\section{Discussion}

The diagnosis of MM is made from a constellation of findings including anemia, monoclonal proteins, bone lesions, renal complications, hypercalcemia, and bone marrow plasmacytosis. These parameters were also used for staging of MM by the Durie-Salmon criteria. The revised international staging system takes the biochemical and cytogenetic profile into account for staging of MM. ${ }^{1}$ Apart from these, many studies have highlighted the role of angiogenesis as an important determinant of prognosis in MM. ${ }^{11-13}$ 


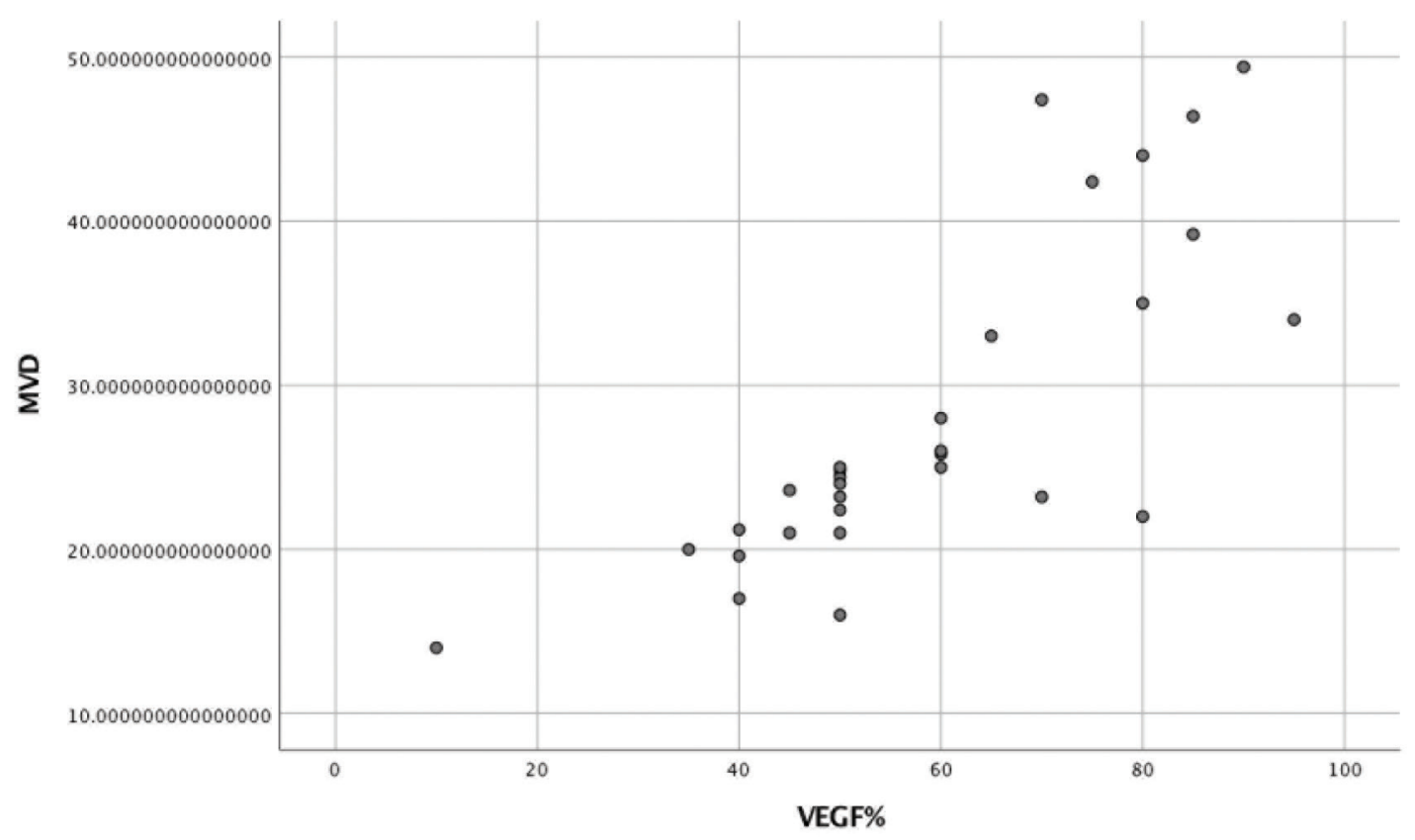

Fig. 3 Correlation between vascular endothelial growth factor (VEGF) expression and microvessel density (MVD).

The concept of angiogenesis in MM is almost three decades old. However, it was debatable if this finding is related to the pathogenesis of the disease or it is merely an epiphenomenon. Most of the recent literature suggests that angiogenesis is an integral part of the pathogenesis of MM that involves the direct production of angiogenic molecules by myeloma cells. ${ }^{14}$ The present study was done to analyze angiogenesis quantitatively by VEGF immunoexpression in plasma cells and by estimating the MVD using CD34 antibody. A comparison has also been drawn between the angiogenic potential of plasmablastic versus nonplasmablastic myeloma.

The mean age of patients with MM in this study was found to be $59.5 \pm 10.32$ years, with slight male preponderance. This is in concordance with the previous studies in literature. . $^{1516}$ The youngest patient in this study was 40 years old. Recent studies in MM have indicated a changing trend with more patients being diagnosed with MM at a younger age. ${ }^{17}$ In the present study, patients with plasmablastic myeloma were older than those with nonplasmablastic MM. However, this difference was not statistically different $(p=0.16)$.

Most of the patients presented with complaints of backache $(56.6 \%)$, generalized weakness (16.6\%), and loss of weight and appetite (13.3\%), all of which are well documented in the literature. ${ }^{9,15,16}$ Radiological examination revealed multiple lytic lesions in $43.3 \%$ of the cases, the percentage in plasmablastic MM and nonplasmablastic MM was 83.3 and $33.3 \%$, respectively. Biochemical tests showed deranged renal function in the form of raised creatinine in $40 \%$ of the cases. The values were higher in plasmablastic myeloma (mean $4.93 \pm 3.87$ ) as compared with nonplasmablastic myeloma (mean $2.01 \pm 1.45$ ), the difference was statistically significant $(p=0.005)$. Also, $\beta 2$ microglobulin levels were raised in all the 30 cases and were found to be significantly higher in plasmablastic as compared with nonplasmablastic category. However, the difference was not statistically significant, which is in contrast to the study by Jakob et al. ${ }^{11}$

Hematological examination revealed anemia in 96.6\% cases, with the average hemoglobin being 7.1 and $8.7 \mathrm{~g} / \mathrm{dL}$ in plasmablastic and nonplasmablastic MM, respectively. The percentage of cases with thrombocytopenia and pancytopenia was higher in plasmablastic group (50 and 16.6\%, respectively) as compared with nonplasmablastic group (12.5 and $4.2 \%$, respectively). Tumor burden (no. of BMPCs) was also significantly higher in plasmablastic MM $(62.33 \pm 13.92)$ compared with nonplasmablastic category ( $43.46 \pm 21.1)$.

Some studies done in the past have correlated plasma cell morphology in bone marrow with the stage of the disease, prognosis, and overall survival. ${ }^{11,15,16}$ In a review of 453 cases of MM (including 36 cases of PB myeloma), Jakob et a ${ }^{11}$ et al found a significantly lower hemoglobin and serum albumin levels, higher calcium and $\beta 2$-microglobuin levels, and higher percentage BMPCs in PB myeloma cases.

VEGF was expressed in all the cases of MM in varying percentage (10-95\%). The mean VEGF expression of $80.83 \pm 7.36$ in plasmablastic myeloma cases was significantly higher compared with a mean VEGF of $53.54 \pm 17.09$ in nonplasmablastic cases. Most of the cases (66.6\%) of plasmablastic myeloma exhibited strong (3+) VEGF expression. A study done by Ribas et $\mathrm{al}^{18}$ found moderate/strong intensity of VEGF staining of plasma cells ( $p=0.036$ ) in PB myeloma. However, an objective measurement of VEGF expression was not provided in their study.

The mean MVD in the present study was $27.933 \pm 9.84$. A positive association was found between plasmablastic morphology and MVD. The mean MVD in plasmablastic cases was $44.8 \pm 3.69$ compared with mean MVD of $23.7 \pm 5.14$ nonplasmablastic category. No association was found between plasmablastic MM and increasing MVD by Ribas et al. ${ }^{18}$ 
The patients in both the categories of MM were treated similarly with a combination therapy comprising melphalan, prednisolone, and thalidomide. However, some of these patients were newly diagnosed and are still under treatment. Therefore, response to treatment between the two groups has not been compared. It could, however, be a subject for further study.

To conclude, we observed a moderate/strong VEGF intensity and higher MVD in cases of plasmablastic MM. This finding suggests that a more aggressive histological disease may be associated with increased production of VEGF and thus increased angiogenesis, which in turn stimulates the production of plasma cells. A correlation between plasmablastic morphology and worsening biochemical and hematological profile has also been found. This finding might be helpful in identifying this subset of patients with adverse prognosis and providing antiangiogenic therapy to improve their survival. However, since the sample size of this study is small, studies comprising larger number of patients are required to bring out a statistical significance to further substantiate these findings.

\section{Sources of support}

None.

\section{Conflict of interest}

None declared.

\section{Acknowledgments}

None.

\section{References}

1 McKenna RW, Kyle RA, Keuhl WM, Harris NL, Coupland RW, Fend F, Plasma cell neoplasms. In: Swerdlow SH, Campo E, Harris NL, Jaffe ES, Pileri Sa, Stein H, eds. WHO Classification of Tumours of Haematopoietic and Lymphoid Tissues. Revised 4th ed. Lyon: IARC; 2017:243-250

2 Greipp PR, Raymond NM, Kyle RA, O'Fallon WM. Multiple myeloma: significance of plasmablastic subtype in morphological classification. Blood 1985;65(2):305-310

3 Xu JL, Lai R, Kinoshita T, Nakashima N, Nagasaka T. Proliferation, apoptosis, and intratumoral vascularity in multiple myeloma: correlation with the clinical stage and cytological grade. J Clin Pathol 2002;55(7):530-534

4 Rajkumar SV, Mesa RA, Fonseca R, et al. Bone marrow angiogenesis in 400 patients with monoclonal gammopathy of undetermined significance, multiple myeloma, and primary amyloidosis. Clin Cancer Res 2002;8(7):2210-2216
5 Podar K, Tai YT, Davies FE, et al. Vascular endothelial growth factor triggers signaling cascades mediating multiple myeloma cell growth and migration. Blood 2001;98(2):428-435

6 GiulianiN,StortiP,BolzoniM,PalmaBD,BonominiS.Angiogenesis and multiple myeloma. Cancer Microenviron 2011;4(3): 325-337

7 Bellamy WT, Richter L, Frutiger Y, Grogan TM. Expression of vascular endothelial growth factor and its receptors in hematopoietic malignancies. Cancer Res 1999;59(3):728-733

8 Kumar S, Fonseca R, Dispenzieri A, et al. Prognostic value of angiogenesis in solitary bone plasmacytoma. Blood 2003; 101(5):1715-1717

9 Bhatti SS, Kumar L, Dinda AK, Dawar R. Prognostic value of bone marrow angiogenesis in multiple myeloma: use of light microscopy as well as computerized image analyzer in the assessment of microvessel density and total vascular area in multiple myeloma and its correlation with various clinical, histological, and laboratory parameters. Am J Hematol 2006;81(9):649-656

10 Greipp PR, Leong T, Bennett JM, et al. Plasmablastic morphology-an independent prognostic factor with clinical and laboratory correlates: Eastern Cooperative Oncology Group (ECOG) myeloma trial E9486 report by the ECOG Myeloma Laboratory Group. Blood 1998;91(7):2501-2507

11 Jakob C, Sterz J, Zavrski I, et al. Angiogenesis in multiple myeloma. Eur J Cancer 2006;42(11):1581-1590

12 Himani B, Meera S, Abhimanyu S, Usha R. Ki-67 immunostaining and its correlation with microvessel density in patients with multiple myeloma. Asian Pac J Cancer Prev 2016;17(5):2559-2564

13 Lee N, Lee H, Moon SY, et al. Adverse prognostic impact of bone marrow microvessel density in multiple myeloma. Ann Lab Med 2015;35(6):563-569

14 Ria R, Berardi S, Reale A, Luisi AD, Catacchio I. Multiple myeloma: the role of angiogenesis in disease progression. J Bone Marrow Res 2013;1:117-119

15 Subramanian R,BasuD, DuttaTK.Prognosticsignificance of bone marrow histology in multiple myeloma. Indian J Cancer 2009; 46(1):40-45

16 Singhal N, Singh T, Singh ZN, Shome DK, Gaiha M. Histomorphology of multiple myeloma on bone marrow biopsy. Indian J Pathol Microbiol 2004;47(3):359-363

17 Costa LJ, Brill IK, Omel J, Godby K, Kumar SK, Brown EE. Recent trends in multiple myeloma incidence and survival by age, race, and ethnicity in the United States. Blood Adv 2017;1(4): 282-287

18 Ribas C, Colleoni GW, Almeida MS, Aguiar KC, Silva MR, Bordin JO. Plasmablastic multiple myeloma is associated with increased vascular endothelial growth factor immunoexpression. Braz J Med Biol Res 2005;38(11):1609-1613 